\title{
Ionization balance for optically thin plasmas: Rate coefficients for all atoms and ions of the elements $\mathrm{H}$ to $\mathrm{Ni}^{\star}$
}

\author{
P. Mazzotta ${ }^{1}$, G. Mazzitelli ${ }^{2}$, S. Colafrancesco ${ }^{3}$, and N. Vittorio ${ }^{1}$ \\ 1 Dipartimento di Fisica, Università di Roma "Tor Vergata", via della Ricerca Scientifica 1, I-00133 Roma, Italy \\ 2 Associazione EURATOM-ENEA sulla Fusione, C.R. Frascati, CP 65-00044 Frascati Roma, Italy \\ 3 Osservatorio Astronomico di Roma, via dell'Osservatorio, I-00040 Monteporzio, Italy
}

Received February 5; accepted June 22, 1998

\begin{abstract}
We present in this paper new and updated calculations of the ionization equilibrium for all the elements from $\mathrm{H}$ to $\mathrm{Ni}$. We collected for these elements all the data available in the literature for the ionization and radiative plus dielectronic recombination rates. In particular, the dielectronic rates have been fitted with a single formula and the related coefficients are tabulated. Our results are compared with previous works.
\end{abstract}

Key words: atomic data - plasmas - radiation mechanisms: thermal — X-rays: general

\section{Introduction}

Hot plasmas are present in the universe in a variety of astrophysical systems, from stellar coronae to the intergalactic medium in clusters of galaxies. The good quality data obtained in the last few decades and up to date from space observatories like EINSTEIN, ROSAT, ASCA and SAX allowed to study in many details the physical condition of several astrophysical plasmas. This activity will have full bloom with the next coming X-ray space missions, like AXAF, XMM and ASTRO-E.

To determine the relevant physical parameters describing both astrophysical plasmas and laboratory plasmas, i.e. electron temperature, density distribution, ion and element abundances, we need to compare observed data with a theoretical spectral model.

Low-density high-temperature astrophysical and laboratory plasmas are generally not in a local thermodynamic equilibrium. To determine the ionization state we

Send offprint requests to: P. Mazzotta

* Tables 1 and 2 are available only in electronic form at the CDS via anonymous ftp to cdsarc.u-strasbg.fr (130.79.128.5) or via http://cdsweb.u-strasbg.fr/Abstract.html need to consider in great details the individual collisional and radiative ionization and recombination processes. For a detailed discussion of the ionization and recombination processes, see e.g. De Michelis \& Mattioli (1981), Mewe (1988, 1990) and Raymond (1988).

In the last two decades various optically thin plasma models have been worked out (see e.g. Raymond \& Smith 1977, Mewe et al. Papers I to VI 1972-1986, Landini \& Monsignori Fossi 1990-1991 (hereafter LM), Sutherland \& Dopita 1993). Generally speaking, the main difference among the various codes in which the most abundant astrophysical elements are considered, lies in the line emission calculations, i.e. both in the number of lines and in the atomic data considered. From the early works up to date there have been extensive improvements in the calculation of atomic parameters and, at the same time, new $\mathrm{X}$-ray observatories are able to get the spectrum of a $\mathrm{X}$-ray source with better and better energy resolution. It comes out the great importance of producing reliable $\mathrm{X}$-ray plasma codes by using the most recent atomic data as well as by improving the algorithms for continuum and line emission processes.

The aim of this work is to compute the ionization balance for the 435 atoms and ions, from $\mathrm{H}(Z=1)$ to $\mathrm{Ni}(Z=28)$, for plasma temperatures in the range $T=0.001 \div 100 \mathrm{keV}$ using the most recent data for the ionization and recombination rates.

Here, we collected all these data making a critical review of the existing works and a detailed comparison among different sources for the available data. In a forthcoming paper (Mazzotta et al. 1998) we will use the ionization equilibrium to compute the continuum and the line radiation emissions.

The plan of the paper is the following: in Sect. 2 we describe briefly the data used for the ionization and radiative recombination rates while in Sect. 3 we discuss, in details, the data adopted for the dielectronic recombination rates that we fitted with a single analytic formula. 
The rate coefficients together with the ionization balance calculations are given in tables. In the last section we compare our results for the ionization equilibrium with those obtained in previous works.

\section{Collisional ionization and radiative recombination rate}

\subsection{Collisional and auto-ionization rate}

To describe the ionization processes we refer to the work of Arnaud \& Rothenflug (1985) and to the updating for the Fe ions of Arnaud \& Raymond (1992) (hereafter AR85 and AR respectively). The contributions to the ionization rates $^{1}$ are given for all the ions of $\mathrm{H}, \mathrm{He}, \mathrm{C}, \mathrm{N}, \mathrm{O}, \mathrm{Ne}$, $\mathrm{Na}, \mathrm{Mg}, \mathrm{Al}, \mathrm{Si}, \mathrm{S}, \mathrm{Ar}, \mathrm{Ca}$ and $\mathrm{Ni}$ from different atomic subshells separately. The direct ionization rate coefficients versus temperature $T$ are given by:

$$
\begin{aligned}
& C_{\mathrm{DI}}=\frac{6.6910^{7}}{(k T)^{3 / 2}} \sum_{j} \frac{\exp \left(-x_{j}\right)}{x_{j}} F\left(x_{j}\right) \quad\left[\mathrm{cm}^{3} / \mathrm{s}\right] \\
& F\left(x_{j}\right)=A_{j}\left[1-x_{j} f_{1}\left(x_{j}\right)\right]+ \\
& +b j\left[1+x_{j}-x_{j}\left(2+x_{j}\right) f_{1}\left(x_{j}\right)\right] C_{j} f_{1}\left(x_{j}\right)+D_{j} x_{j} f_{2}\left(x_{j}\right) .
\end{aligned}
$$

The summation is performed over the subshells $\mathrm{j}$ of the ionizing ion with

$$
\begin{aligned}
& x_{j}=\frac{I_{j}}{k T}, \quad f_{1}(x)=\mathrm{e}^{x} \int_{1}^{\infty} \frac{\mathrm{d} t}{t} \mathrm{e}^{-t x}, \\
& f_{2}(x)=\mathrm{e}^{x} \int_{1}^{\infty} \frac{\mathrm{d} t}{t} \mathrm{e}^{-t x} \ln (t),
\end{aligned}
$$

and where $k T$ and $I_{j}$ are in $\mathrm{eV}$. The values parameters $A_{j}, B_{j}, C_{j}$ and $D_{j}$ are given in AR85 and AR. Following AR85 and AR we also take into to account the excitationautoionization (hereafter EA) contribution of ions in the ground state. This is a good approximation for low-density plasmas (as in supernova remnants or clusters of galaxies) because the lifetime of excited states is small as compared to the mean collision time. The other ions not included in the AR85 work are calculated by interpolation or extrapolation along the isosequence.

\subsection{Radiative recombination rate}

For the radiative recombination rates $^{1}$ the calculations of Shull \& Van Steenberg (1982) (hereafter SV) were used for some of the most abundant astrophysical elements $(\mathrm{Mg}$, $\mathrm{Si}, \mathrm{S}, \mathrm{Ar}, \mathrm{Ca}, \mathrm{Fe}, \mathrm{Ni})$. They give the fitting parameters $A$ and $\eta$ for the following formula:

$\alpha_{\mathrm{r}}=A\left(\frac{T}{10^{4} \mathrm{~K}}\right)^{\eta} \quad\left[\mathrm{cm}^{3} / \mathrm{s}\right]$,

\footnotetext{
1 The data are available in electronic form at the following web address:

http://www.pa.uky.edu/ verner/fortran.html
}

with the electron temperature $T$ in $\mathrm{eV}$. LM extrapolated these calculations also to other astrophysical less abundant elements. For Fe XV-Fe XXIV we used the formula of AR:

$\alpha_{\mathrm{r}}=A\left(\frac{T}{10^{4} \mathrm{~K}}\right)^{\alpha-\beta \log _{10}\left(\frac{T}{10^{4} \mathrm{~K}}\right)} \quad\left[\mathrm{cm}^{3} / \mathrm{s}\right]$,

where the electron temperature $T$ is in Kelvin and the fitting parameters $A, \alpha, \beta$ are given by $\mathrm{AR}$ in tabular form. For the H-like, He-like, Li-like and Na-like isosequences we used the new calculations, in the framework of the opacity project, of Verner \& Ferland (1996). They fit the data by the following formula:

$\alpha_{\mathrm{r}}=A\left[\sqrt{\frac{T}{T_{0}}}\left(1+\sqrt{\frac{T}{T_{0}}}\right)^{1-b}\left(1+\sqrt{\frac{T}{T_{1}}}\right)^{1+b}\right]^{-1}$

$$
\left[\mathrm{cm}^{3} / \mathrm{s}\right],
$$

with the electron temperature $T$ in Kelvin, and the fitting parameters $A, b, T_{0}, T_{1}$ are given for all the ions from $\mathrm{H}$ through $\mathrm{Zn}$. This last formula is valid in a wide range of temperatures, from $T=2.510^{-4} \mathrm{eV}$ to $T=100 \mathrm{keV}$. We also use the same formula for $\mathrm{C}, \mathrm{N}, \mathrm{O}$ and Ne ions with the fitting parameters of Péquignot et al. (1991).

\section{Dielectronic recombination rate}

Burgess (1965) pointed out the relevance of the dielectronic recombination (hereafter DR) process especially in the temperature region of maximum ion abundance. The collected dielectronic recombination rates are calculated in the zero electron density limit, which is a valid approximation for most of the astrophysical plasmas.

In the following, as in AR, we discuss the dielectronic recombination rates by isosequence and we explicitly declare when we have chosen different data from them.

All the available data were fitted, when needed, with the following formula:

$\alpha_{\mathrm{d}}=\frac{1}{T^{3 / 2}} \sum_{j=1}^{4} c_{j} \exp \left(-\frac{E_{i}}{T}\right) \quad\left[\mathrm{cm}^{3} / \mathrm{s}\right]$,

where $T$ and $E_{i}$ are given in $\mathrm{eV}$ and $c_{i}$ in $\mathrm{cm}^{3} \mathrm{~s}^{-1}$. The coefficients $c_{i}$ and $E_{i}$ are given in Table 1.

H-like - From $\mathrm{C}$ to $\mathrm{Ni}$ we used the data of Mattioli 1988 (hereafter M) and Karim \& Bhalla (1988)which for the same elements are in good agreement. For the elements not explicitly calculated we interpolated the DR rates along the isosequence. For Be IV and B V we used the data of Pindzola \& Badnell (1992) that are a factor 2 less then those from previous calculations of Shore (1969) with the corrected values by Burgess \& Tworkowski (1976). Moreover we divided by a factor 2 also the DR coefficients for HeII and Li III by Shore (1969).

He-like - We fitted with our formula the calculations of Chen (1986a) that are in very good agreement with Nielsen (1986) and Karim \& Bhalla (1989). For C V we 
adopt Chen's (1988b) calculation in which the CosterKroning transitions are taken into account that are negligible for higher $\mathrm{Z}$ elements. For BeIII and B IV we adopted Pindzola \& Badnell (1992).

Li-like - We adopt the DR calculations of Chen (1991). The total DR rate coefficient for the 11 lithium-like ions were calculated using the distorted wave technique and the multi-configuration Dirac-Fock method. The discrepancies with non-relativistic calculations of Roszman (1987) are more relevant for highly charged medium- and high-Z ions. For the other ions we have interpolated along the isosequence. For BeII and B III we have adopted the DR calculations of Pindzola \& Badnell (1992).

Be-like - We adopt Badnell's (1987) calculations which for high Z agree well with Chen \& Crasemann (1988). For O V and B II we use, respectively, the data of Badnell \& Pindzola (1989) and Pindzola \& Badnell (1992).

B-like - Recent calculations of Nahar (1995) and errata from Nahar (1996a), in the framework of a unified treatment of electron-ion recombination, show good agreement with low-T DR rates by Nussbaumer \& Storey (1983), and high-T DR rates by Jacobs et al. (1979) for the ions from $\mathrm{C}$ to $\mathrm{Al}$, and for $\mathrm{Si}$ and $\mathrm{S}$. As for $\mathrm{Fe}$, we use the data of AR. For most of the other ions calculations from SV, LM and M are available. For the other ions we used the Burgess (1965) general formula, corrected by Merts et al. (1976) (hereafter Burgess-Merts formula, BM), using the most recent data for oscillator strengths and energy transitions.

Iron is the most investigated element so that in the following we always adopt the criteria, except when more recent calculations are available, to normalize the DR calculation rates to the Fe calculations of AR. These criteria can be also justified by the fact that, as pointed out in LM (1991) and Hahn (1991), most of the coefficients show a regular trend along the same isosequence.

$C$-like - N-like - From Ar to $\mathrm{Ni}$ we followed the same procedure described for B-like ions. For the other elements, except for O III for which we used the data from Badnell \& Pindzola (1989), we used the data of Jacobs et al. $(1977 \mathrm{a}, \mathrm{b}, 1979,1980)$ and LM (1991).

O-like - F-like - From Ar to Ni we adopt the recent calculations of Dasgupta \& Whitney $(1990,1994)$ that for F-like ions are in good agreement with Chen (1988a) calculations. For the other elements we used the calculations of SV.

Ne-like - We use the calculations of Chen (1986b), Romanik (1988) and for Na II the calculation of SV. For P VI and Cl VIII we adopted the data of LM(1991) multiplied by a factor 4 to take into to account the results of Romanik (1988) and Chen (1986b) for adjacient ions.

Na-like - Mg-like - As it has been indicated by Zhdanov (1982), Dube \& LaGatutta (1987), Dube et al. (1985), at high temperature the inner shell transitions of the form $2 \mathrm{~s}-3 \mathrm{~d}$ become important, and this effect is more important for medium and high-Z elements. We follow the procedure of Mattioli (1988) that for Fe is in very good agreement with AR. For the other elements we adopt the calculations of Jacobs and LM taking into account the results obtained for $\mathrm{Mg}$ II and Si IV from LaGattuta \& Hahn $(1982,1984)$. For S VI we adopt the data of Badnell (1991).

Al-like - to Co-like - For all these isosequences, except for $\mathrm{Fe}$, detailed or recent calculations are not available so that we used data from SV, LM, M as well as the general $\mathrm{BM}$ formula. Up to the Mn-like isosequence we renormalize the results to the work of AR.

We mention that recently new calculations have been made on the recombination of Fe II (Nahar et al. 1997) and Fe IV (Nahar 1996b). In the case of Fe II the DR rate is a factor 2 above the AR curve whereas for Fe IV the Nahar results are an order of magnitude below the AR curve although Nahar claims a $10-30 \%$ accuracy. We do not know the cause of the discrepancies but for consistency we prefer to use the AR results. In the last years Teng et al. (1994a-d, 1996) also applied fitting formula for several isoelectronic sequence i.e. $\mathrm{H}, \mathrm{He}, \mathrm{Li}, \mathrm{F}$ and Ne. In general our results are within a $10 \%$ in agreement with those calculations essentially because we have fitted and/or interpolated the same data, but in some case their fitting formula yield divergences in the dielectronic rates.

\section{Results for the coronal equilibrium}

In Table 2 we give the ionization fraction $X_{\mathrm{i}}$, for the 28 elements. In these calculations we included only the collisional processes in the low-density case. We compared our ionic abundances fractions with those given by AR85 (AR for the iron). We found a very good agreement or differences less then $10 \%$ for all the atoms and ions of $\mathrm{H}, \mathrm{He}$, $\mathrm{N}, \mathrm{Ne}, \mathrm{Na}, \mathrm{Mg}$, Si. For C II, C III, O III, O IV, O V we found differences up to $50 \%$ near the peaks of maximum ionic abundance, but good agreement for the other ions of these elements. In Fig. 1 we compare our ionic fraction for the less ionized ions of $\mathrm{Al}$ with those of AR85. In the same figure we report, for each ion, also the percent variation near the peak of maximum ionic abundance. We can note that our ionic abundance fraction at $T \approx 210^{5} \mathrm{~K}$ is a factor of 2 greater than those of AR85. In Figs. 2 to 4 we report the comparisons for some $\mathrm{Ar}$ and $\mathrm{Ca}$ ions. In those cases we found, depending on the temperature and on the ions considered, differences up to $400 \%$. The curves obtained for the iron ions are both or the same or in very good agreement with those of AR except for the ions from Fe XVII to Fe XXI due to the updating of Fe XIX dielectronic recombination rate, as shown by Fig. 5, where we still have small variations. In Figs. 6 to 9 we compare the curves for some Ni ions and we found substantially differences with respect to AR85. If we fix the temperature at which the ion abundance curves reach their maxima, we can see that generally the big differences found are due to 


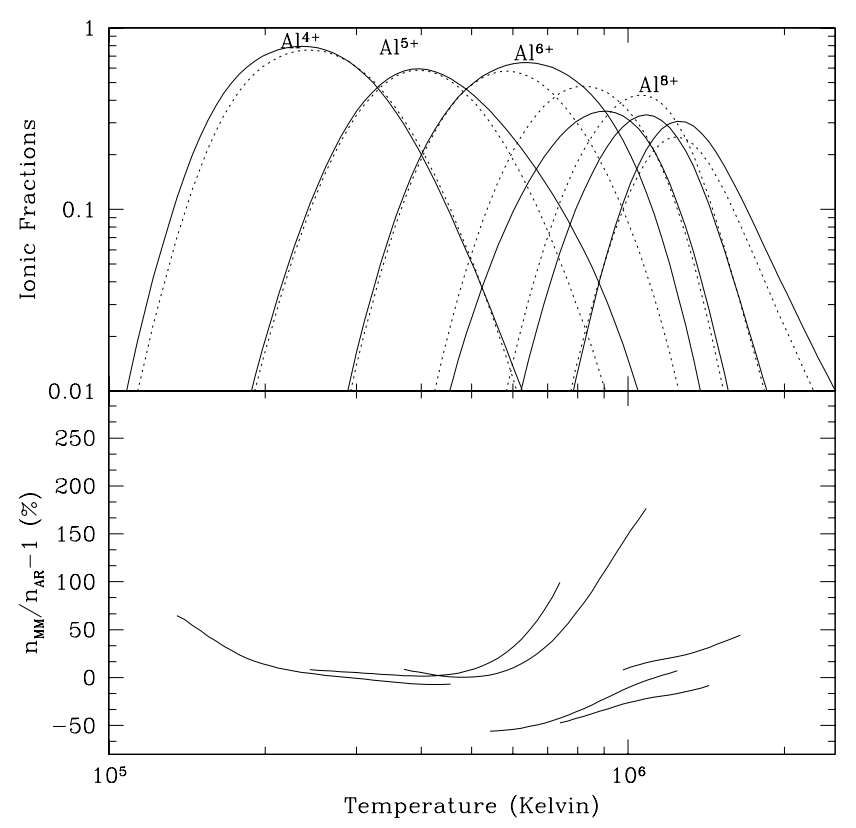

Fig. 1. Upper panel: Ionic fraction vs. temperature for $\mathrm{Al}$ ions, from $\mathrm{Al} \mathrm{V}$ to $\mathrm{Al} \mathrm{X}$. Solid curves: present work; dashed curves: AR85. Lower panel: percent variations in the ionic abundance fractions in the present work with respect to that of AR85. For each ion, the percent variations are evaluated only for a range of temperatures in which the respective ionic fractions are $>10^{-1}$

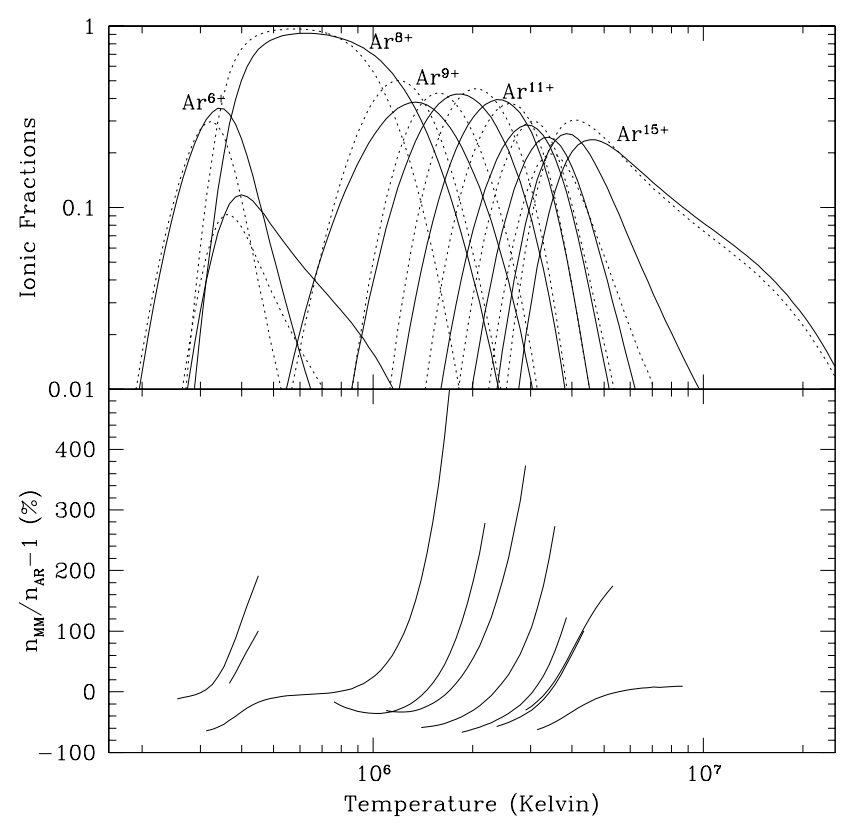

Fig. 2. Same as Fig. 1 but for Ar ions, from Ar VII to Ar XVI

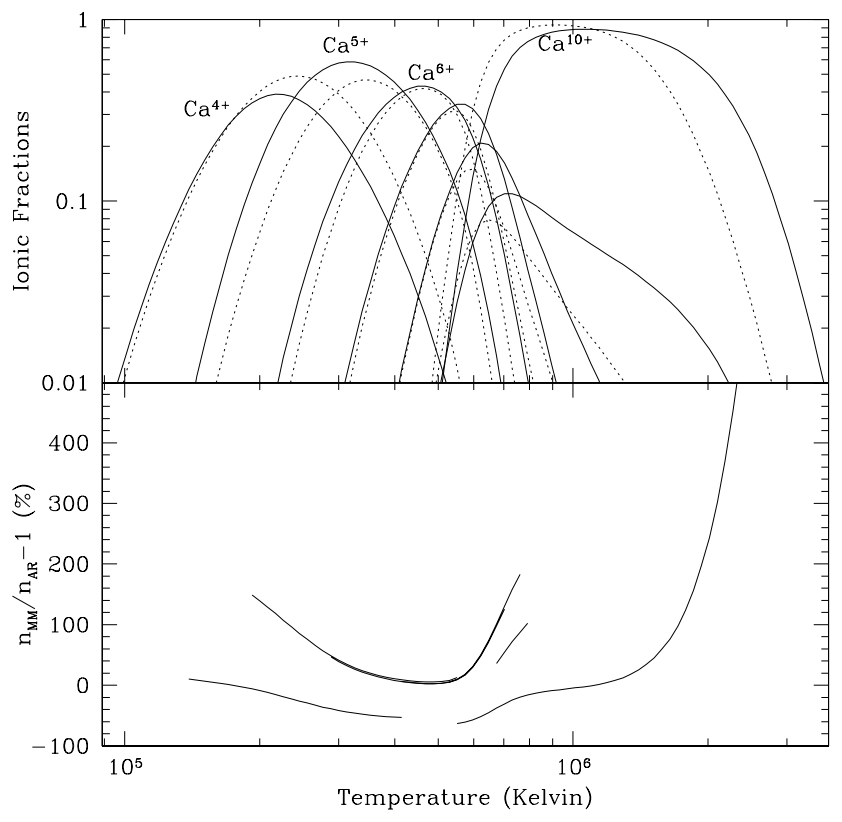

Fig. 3. Same as Fig. 1 but for Ca ions, from Ca V to Ca XI

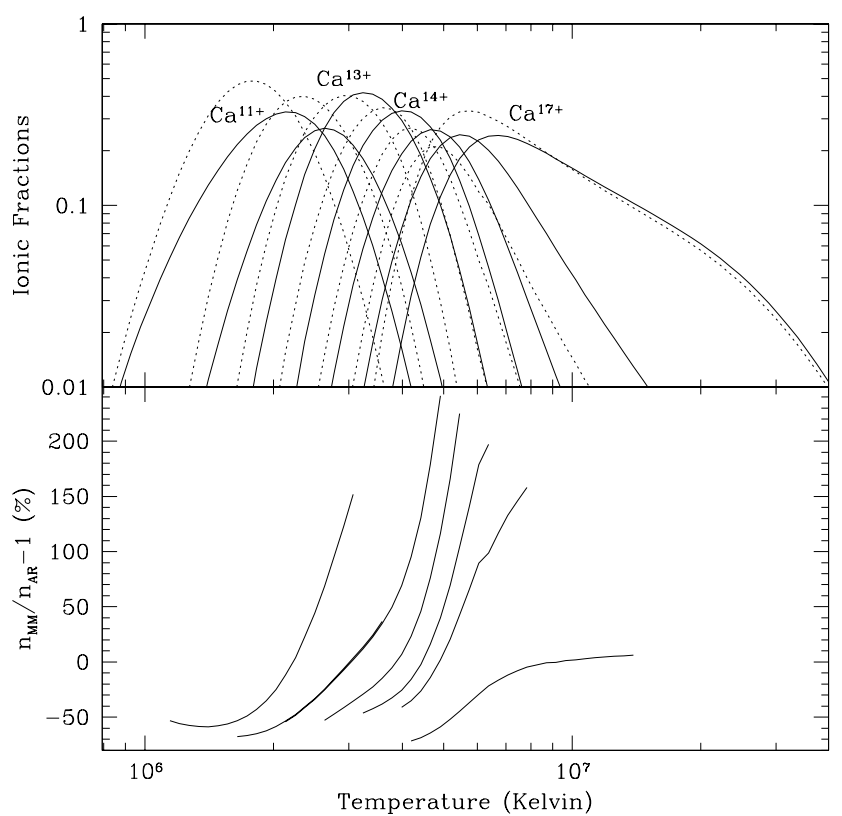

Fig. 4. Same as Fig. 1 but for Ca ions, from Ca XII to Ca XVIII

a shift, depending on the considered ion, to higher or lower temperature with respect to the other considered case.

We compered, also, our ionic abundance curves for the less abundant astrophysical elements with those of Landini \& Monsignori Fossi (1991) and found again significant differences, depending on the temperature and ion considered. In Fig. 10, as an example, we show the comparison of our ionic fraction curves for the most ionized ions of Cr with those of Landini \& Monsignori Fossi (1991) 


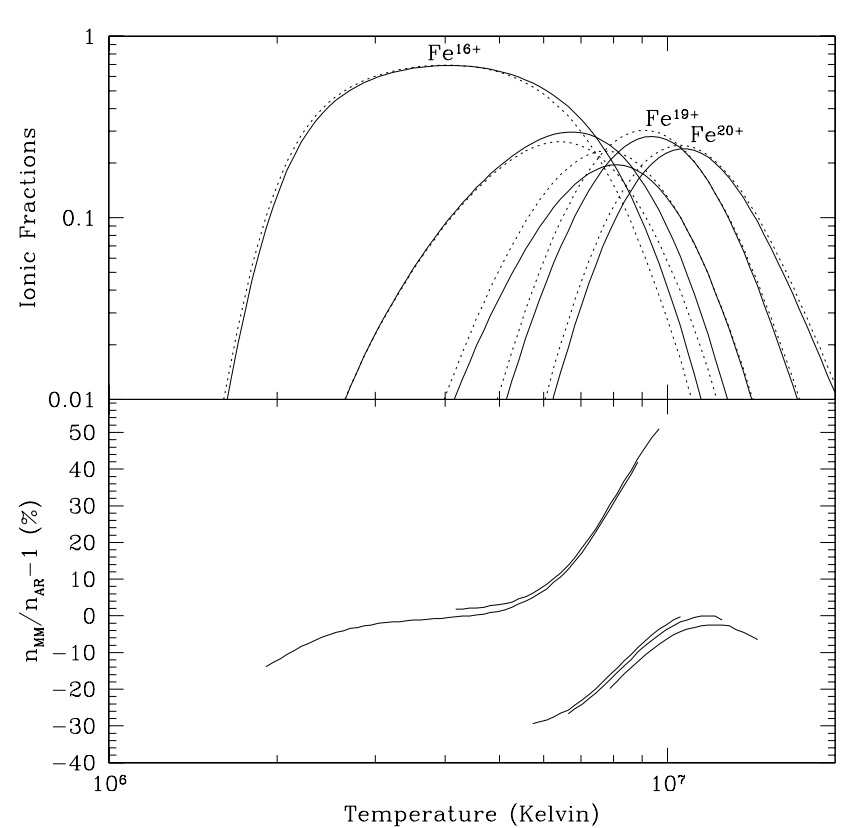

Fig. 5. Upper panel: Ionic fraction vs. temperature for Fe ions, from Fe XVII to Fe XXI. Solid curves: present work; dashed curves: AR. Lower panel: percent variations in the ionic abundance fractions in present work with respect to AR. For each ion the percent variations are evaluated only for a range of temperatures in which the respective ionic fractions are $>10^{-1}$

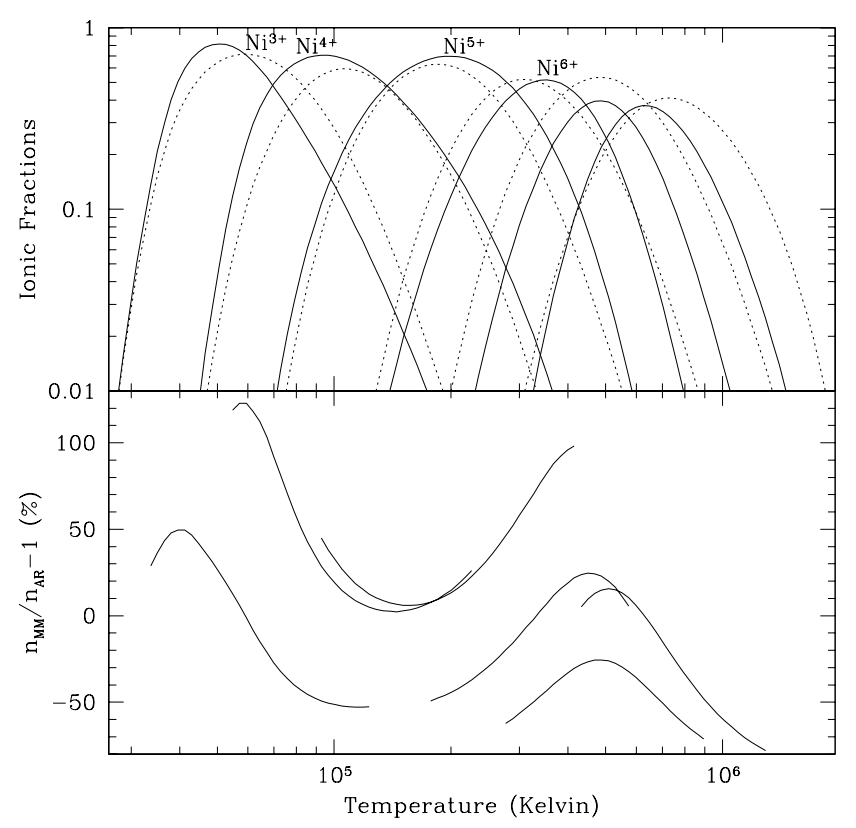

Fig. 6. Same as Fig. 1 but for Ni ions, from Ni IV to Ni IX

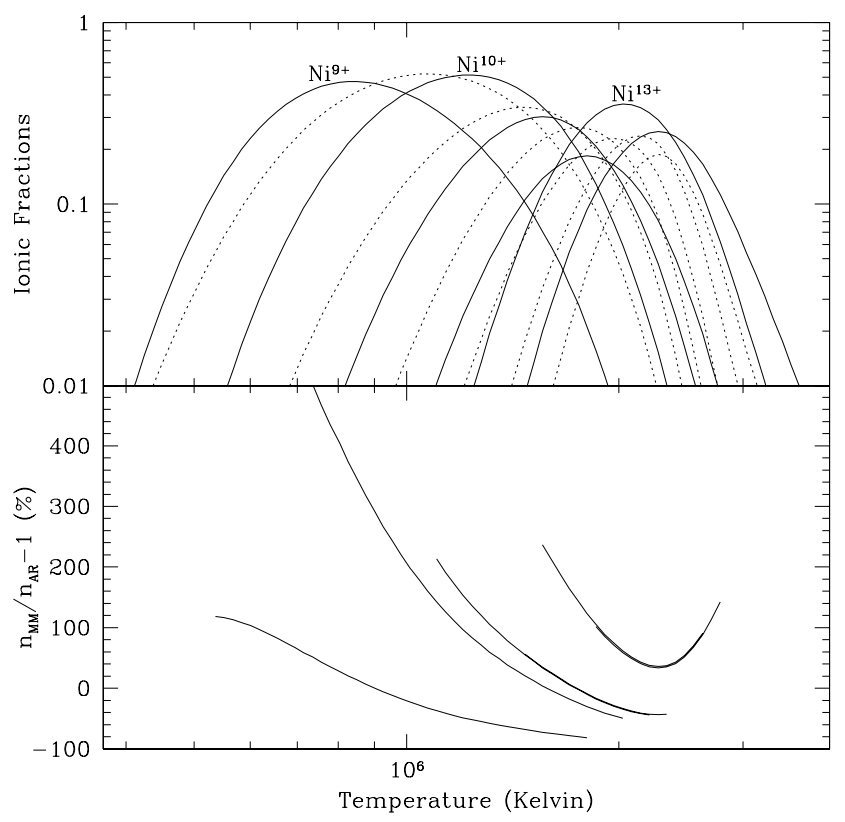

Fig. 7. Same as Fig. 1 but for Ni ions, from Ni X to Ni XV

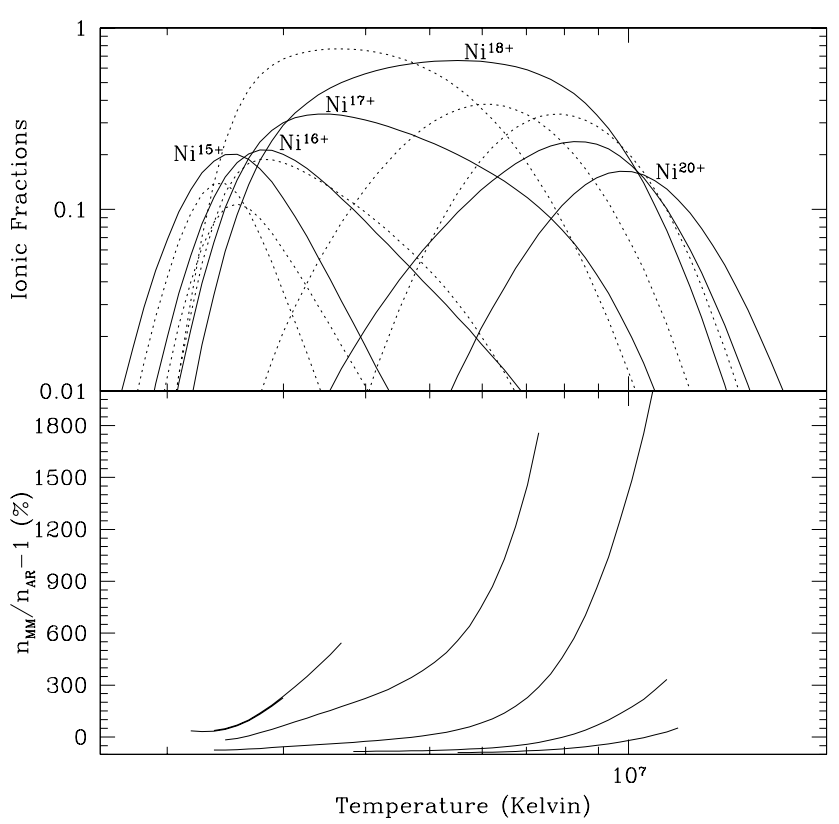

Fig. 8. Same as Fig. 1 but for Ni ions, from Ni XVI to Ni XXI

and we can observe that our curves are shifted to higher temperatures.

\section{Discussion}

The main goal of this work was also to collect all available data on the collisional ionization, radiative and dielectronic recombination rates data. Most of this data 


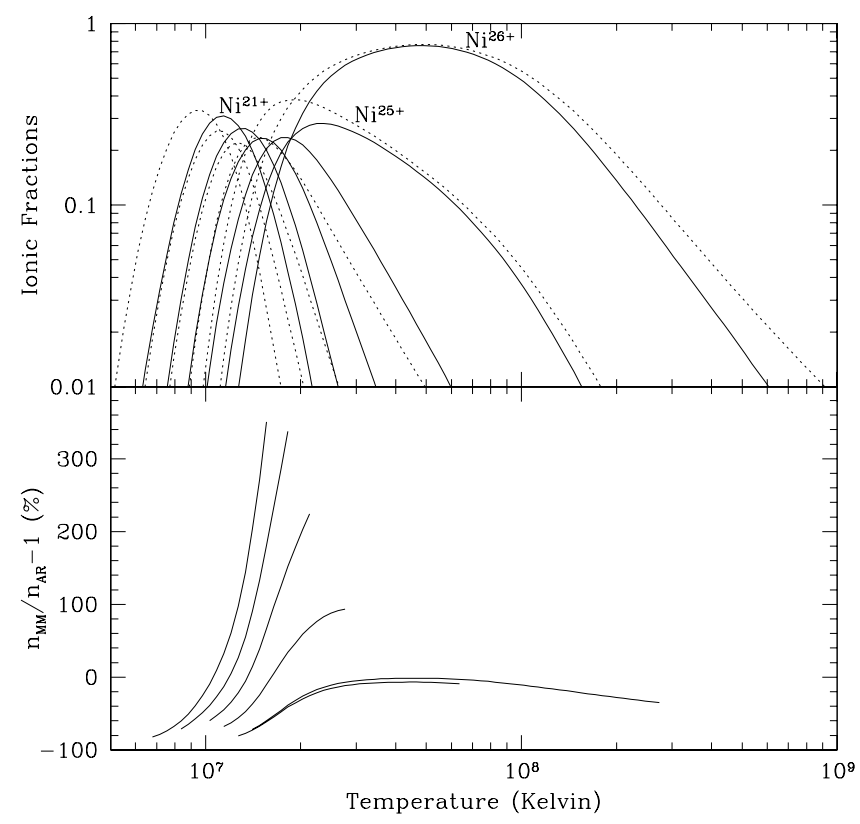

Fig. 9. Same as Fig. 1 but for Ni ions, from Ni XXII to Ni XXVII

come out from new advanced computational calculations. About the ionization rates we would like to remark that recently Kato et al. (1991) reviewed and compared the rates obtained with several empirical formulae used by Lotz (1967, 1968), by the Belfast group (Bell et al. 1983 and Lennon et al. 1988), by AR85 and AR, and by Pindzola et al. (1987). They conclude that generally the rate coefficient derived on the basis of experimental data of cross sections are in good agreement among the various authors. Otherwise when no experimental data are available only AR85 and AR included the EA process. This reflects in a difference at high temperature for some isosequences (e.g., Na-like, Li-like, and Mg- to Ar-like sequences). Kato et al. (1991) also remark that for some ions the Belfast group rates are lower by a factor 1.626 due to a misprint on the Younger (1982) table. Based on the Kato results, Voronov (1997) refitted the data of the Belfast group with a very simple formula for the total ionization rates. Instead of its simplicity we do not use the Voronov data for the following reason: i) Voronov give a fit only for the total ionization rate. This formula is valid as long as we consider the case of ionization equilibrium plasmas. Under nonequilibrium conditions (such as apply to transient plasmas which are present e.g., in supernova remnants and solar/stellar flares) inner-shell ionization may play an important role, both in the determination of the ionization balance and in the formation of fluorescent lines. Then it can be important in certain cases (e.g., for the Be- and B-like sequences) to know the contribution from different atomic subshells separately (Mewe private communication). ii) we checked the Voronov results and we saw that, instead of his claim, some curves are in agreement

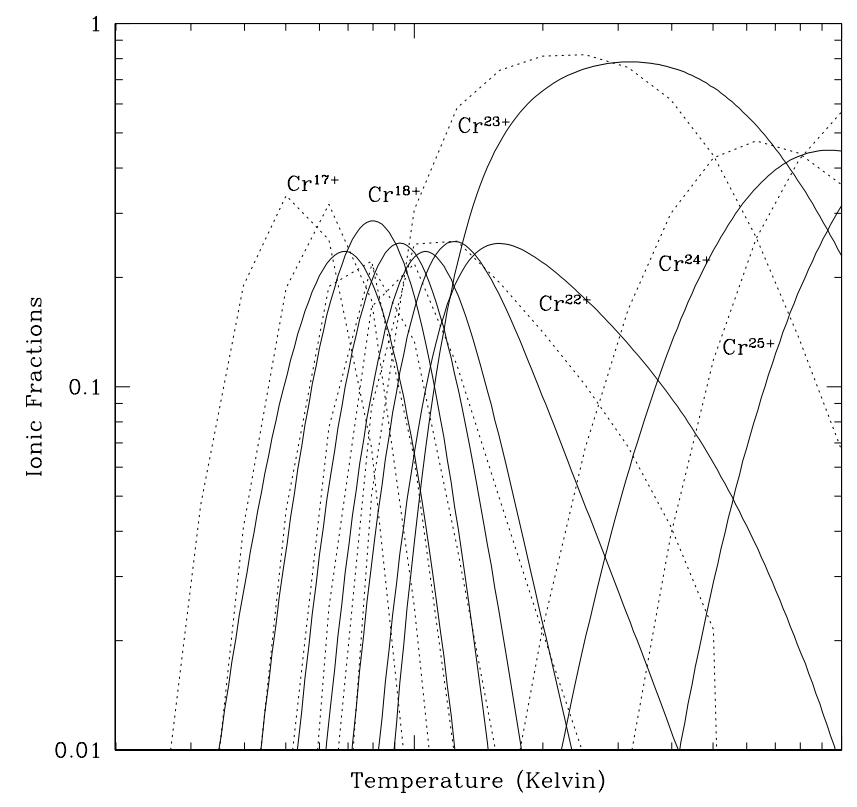

Fig. 10. Ionic fraction vs. temperature $\mathrm{Cr}$ ions, from Cr XVIII to Cr XXVI. Solid curves: present work; dashed curves: Landini \& Monsignori Fossi (1991)

with the original data of the Belfast group without the correction factor of 1.626 (which implies that in these cases his results are just a factor of 1.626 below the corresponding AR results, see e.g. Kato et al. (1991) for Fe XXII to Fe XXVI). So, for some ions, the Voronov ionization rates are uncorrected. We would like to remark that the ionization rates for $\mathrm{Ni} \mathrm{I}$ to $\mathrm{Ni} \mathrm{X}$ are probably wrong by large factors due to a large underestimate of the Excitation autoionization process in the AR85 (Arnaud private communication). In a forthcoming paper (Kaastra et al. 1998) we intend to update the ionization rate including EA, resonance excitation double autoionization and direct multiple ionization.

We believe that at present our atomic data collection represents the state of the art in such calculations. An important point of this work was to reorganize all the available data with a few and simple fitting formulae in order to use them in a numerical modeling of plasma processes.

Obviously, the differences in the ionization fraction between our work and the previous ones, reflects directly in the computed line power emissions. In particular, our preliminary calculations show sensitive differences, depending on the selected plasma temperature, in the computation of the total line radiation emission with respect to previous similar work. We will fully address these issues in a forthcoming paper.

Acknowledgements. We are grateful to R. Mewe, for helpful comments and suggestions. We are indebted to M. Mattioli who has pointed out to us some mistakes in the data. 


\section{References}

Arnaud M., Rothenflug R., 1985, A\&AS 60, 425 (AR85)

Arnaud M., Raymond J., 1992, ApJ 398, 394 (AR)

Badnell N.R., 1987, J. Phys. B 20, 2081

Badnell N.R., Pindzola M.S., 1989, Phys. Rev. A 39, 1690

Badnell N.R., 1991, ApJ 379, 356

Bell K.L., Gilbody H.P., Hughes J.G., Kingston A.E., Smith F.J., 1983, J. Phys. Chem. Ref. Data 12, 891

Burgess A., 1965, ApJ 141, 1588 (BM)

Burgess A., Tworkowski A.S., 1976, ApJ 205, L105

Chen M.H., 1986a, Phys. Rev. A 33, 1986

Chen M.H., 1986b, Phys. Rev. A 34, 1073

Chen M.H., 1988a, Phys. Rev. A 38, 2332

Chen M.H., 1988b, Phys. Rev. A 38, 6430

Chen M.H., Crasemann B., 1988, Phys. Rev. A 38, 5595

Chen M.H., 1991, Phys. Rev. A 44, 4215

Dasgupta A., Whitney K.G., 1990, Phys. Rev. A, 42, 2640

Dasgupta, A., Whitney K.G., 1994, ADNDT, 58, 77

De Michelis C., Mattioli M., 1981, Nuclear Fusion 21, 677

Dube M.P., Rosoanaivo R., Hahn Y., 1985, JQSRT 33, 13

Dube M.P., LaGatutta K.J., 1987, JQSRT 38, 311

Gronenschild E.H.B.M., Mewe R., 1978, A\&AS 32, 283 (Paper III)

Hahn Y., 1991, Phys. Scr. T37, 53

Jacobs V.L., Davis J., Kepple P.C., Blaha M., 1977a, ApJ 211, 605

Jacobs V.L., Davis J., Kepple P.C., Blaha M., 1977b, ApJ 215, 690

Jacobs V.L., Davis J., Rogerson J.E., Blaha M., 1979, ApJ 230, 627

Jacobs V.L., Davis J., Rogerson J.E., Blaha M., Cain J., Davis M., 1980, ApJ 239, 1119

Kaastra J.S., et al., 1998 (in preparation)

Karim K.R., Bhalla C.P., 1988, Phys. Rev. A 37, 2599

Karim K.R., Bhalla C.P., 1989, Phys. Rev. A 39, 3548

Kato T., Masai K., Arnaud M., 1991, NIFS-DATA-14

LaGattuta K.J., Hahn Y., 1982, J. Phys. B 15, 2101

LaGattuta K.J., Hahn Y., 1984, Phys. Rev. A 30, 316

Landini M., Monsignori Fossi B.C., 1990, A\&AS 82, 229 (LM)

Landini M., Monsignori Fossi B.C., 1991, A\&AS 91 (LM), 183

Lennon M.A., Bell K.L., Gilbody H.B., Hughes J.G., Kingston A.E., Murray M.J., Smith F.J., 1988, J. Phys. Chem. Ref. Data 17,1285

Lotz W., 1967, Z fur Phys. 206, 205

Lotz W., 1968, Z fur Phys. 216, 241

Mattioli M., 1988, Report EUR-CEA-FC, 1346 (M)

Mazzotta P., Mazzitelli G., Colafrancesco S., Vittorio N., 1998 (in preparation)

Merts A.L., Cowan R.D., Magee J., 1976, Los Alamos Scientific Laboratory Report La-6220-MS (BM)

Mewe R., 1972, Solar Phys. 22, 459 (Paper I)

Mewe R., 1975, Solar Phys. 44, 383 (Paper II)

Mewe R., 1988, in Astrophysical and Laboratory Spectroscopy, Brown R., Lang J. (eds.). Scottish Univ. Summer School in Phys. Publ., p. 129

Mewe R., 1990, in Physical Processes in Hot Cosmic Plasmas, Brinkmann W., Fabian A.C., Giovanelli F. (eds.). Kluwer Acad. Publ., Dordrecht-Holland, p. 39

Mewe R., Gronenschild E.H.B.M., 1981, A\&AS 45, 11 (Paper IV)

Mewe R., Gronenschild E.H.B.M., van den Oord G.H.J., 1985, A\&AS 62, 197 (Paper V)

Mewe R., Lemen J.R., van den Oord G.H.J., 1986, A\&AS 65, 551 (Paper VI)

Nahar S.N., 1995, ApJS 101, 423

Nahar S.N., 1996a, ApJS 106, 213

Nahar S.N., 1996b, Phys. Rev. A 53, 2417

Nahar N., Bautista M.A., Pradhan A.K., 1997, ApJ 479, 497

Nielsen J., 1986, J. Phys. B 19, 2401

Nussbaumer H., Storey P.J., 1983, A\&A 126, 75

Péquignot D., Petitjean P., Boisson C., 1991, A\&A 251, 680

Pindzola M.S., Griffin D.C., Bottcher C., Younger S.M., Hunter H.T., 1987, Nucl. Fusion, Special Supp., 21

Pindzola M.S., Badnell N.R., 1992, Nucl. Fusion and Plasma Material Interaction data for fusion Vol. 3, 101

Raymond J.C., 1988, in Hot Thin Plasmas in Astrophysics, Pallavicini R. (ed.). Kluwer Acad. Publ., DordrechtHolland, p. 3

Raymond J.C., Smith B.W., 1977, ApJS 35, 419

Romanik C.J., 1988, ApJ 330, 1022

Roszman L.J., 1987, Phys. Rev. A 35, 2122

Shore B.W., 1969, ApJ 158, 1205

Shull J.M., Van Steenberg M., 1982, ApJS 48, 95 (SV)

Sutherland R.S., Dopita M.A., 1993, ApJS 88, 253

Teng H., Sheng B., Zhang W., Xu Z., 1994a, Phys. Scr. 49, 463

Teng H., Sheng B., Zhang W., Xu Z., 1994b, Phys. Scr. 49, 468

Teng H., Sheng B., Zhang W., Xu Z., 1994c, Phys. Scr. 49, 696

Teng H., Sheng B., Zhang W., Xu Z., 1994d, Phys. Scr. 55, 55

Teng H., Xu Z., 1996, J. Quant. Spectrosc. Radiat. Transfer 56,443

Verner D.A., Ferland G.J., 1996, ApJS 103, 467

Voronov G.S., 1997, ADNDT65, 1

Younger S.M., 1982, JQRST 27, 541

Zhdanov V.P., 1982, J. Phys. B 15, L537 